\title{
6 Zusammenfassung
}

In dieser Arbeit wurden Möglichkeiten untersucht, um Unsicherheiten im Entwurf modellprädiktiver Regler für nichtlineare Systeme zu berücksichtigen. Neben dem Überblick über bestehende Ansätze aus der Literatur wurde ein neues Verfahren auf Grundlage der Unscented-Transformation erarbeitet. Der Ansatz unterscheidet sich von den bestehenden dadurch, dass er auf der zeitkontinuierlichen Systemdarstellung basiert. Des Weiteren kann das dynamische Optimierungsproblem für den Unscented-Ansatz mit einem suboptimalen Gradientenverfahren gelöst werden und erlaubt damit eine effiziente Implementierung. Die bestehende Software GRAMPC wurde um das Verfahren erweitert, so dass direkte Vergleiche mit der deterministischen modellprädiktiven Regelung möglich sind.

Bei der Evaluation anhand von einem Rührkesselreaktor und einem Laborkran wurde gezeigt, dass die Zustandsbeschränkungen mit dem Unscented-Ansatz auch in Gegenwart von stochastischen Unsicherheiten sicher eingehalten werden können. Bei hohem Messrauschen hat sich der zusätzliche Einsatz eines Zustandsschätzers bewährt. Wenn die beiden Systeme ohne Beschränkungen betrachtet werden, dann kann der stochastische Ansatz keine nennenswerte Verbesserung der Kosten erzielen.

Darüber hinaus wurde der Rechenaufwand des Unscented-Ansatzes für ein skalierbares System aus Feder-Masse-Dämpfern untersucht. Dabei wurde gezeigt, dass die Gesamtzeit für ein System mit $n$ Differentialgleichungen in der Größenordnung $\mathcal{O}\left(n^{5}\right)$ liegt. Die Laufzeit ist zwar polynomiell, aber für große Systeme, wie sie z. B. bei der Diskretisierung von partiellen Differentialgleichungen entstehen, ist der Ansatz damit nicht einsetzbar.

Ein Problem ist während den Tests an verschiedenen Systemen wiederholt aufgetreten. Für die Cholesky-Zerlegung muss die Kovarianzmatrix stets positiv definit sein. In mehreren Fällen ist die Matrix im Lauf der Integration jedoch indefinit geworden, so dass das Verfahren abgebrochen werden musste. Als Gegenmaßnahme kann ein genaueres Integrationsverfahren, wie z. B. Runge-Kutta-Verfahren, eingesetzt oder die Anzahl der Stützstellen erhöht werden. Wenn das Problem entsteht, sobald die Kovarianz gegen Null geht, dann kann die Systemunsicherheit absichtlich erhöht werden, so dass die Einträge auf der Diagonalen die positive Definitheit gewährleisten. Bei instabilen Systemen, bei denen die Kovarianz unbeschränkt wächst, kann das Problem als Hinweis verstanden werden, dass der Zeithorizont zu lange ist. Da die Prädiktion in diesem Fall unzuverlässig ist, sollte besser ein kurzer Horizont gewählt werden.

Weitere Möglichkeiten für Untersuchungen bieten sich vor allem beim Vergleich des Unscented-Verfahrens mit anderen Ansätzen aus der Literatur und bei der Evaluation von weiteren Systemen. Hier scheint es bisher kein etabliertes Benchmark-Beispiel für die Regelung mit Unsicherheiten zu geben. Schließlich ist die Erprobung an einem Versuchsaufbau, bei dem Unsicherheiten ein reales Problem darstellen, von besonderem Interesse. 